Article

\title{
The Class Equation and the Commutativity Degree for Complete Hypergroups
}

\author{
Andromeda Cristina Sonea ${ }^{1, *(1)}$ and Irina Cristea $2, *$ (D) \\ 1 Faculty of Mathematics, Al. I. Cuza University, Bd. Carol I 13, 700506 Iasi, Romania \\ 2 Centre for Information Technologies and Applied Mathematics, University of Nova Gorica, \\ Vipavska Cesta 13, 5000 Nova Gorica, Slovenia \\ * Correspondence: cristina.sonea@uaic.ro (A.C.S.); irina.cristea@ung.si (I.C.); Tel.: +386-0533-15-395 (I.C.)
}

Received: 19 November 2020; Accepted: 17 December 2020; Published: 21 December 2020

\begin{abstract}
The aim of this paper is to extend, from group theory to hypergroup theory, the class equation and the concept of commutativity degree. Both of them are studied in depth for complete hypergroups because we want to stress the similarities and the differences with respect to group theory, and the representation theorem of complete hypergroups helps us in this direction. We also find conditions under which the commutativity degree can be expressed by using the class equation.
\end{abstract}

Keywords: complete hypergroup; commutativity degree; conjugacy class; class equation

\section{Introduction}

In a non-abelian group-or, more generally, in a non-abelian algebraic structure-it makes sense to compute the probability that two elements commute. This problem was first addressed by Miller [1] in 1944, when he studied the relative number of non-invariant operators in a group. Later on, Erdös and Turan [2] introduced the concept of commutativity degree as the probability that an arbitrary element $x$ in a finite group $G$ commutes with another arbitrary element $y$ in $G$, that is, $d(G)=|\{(x, y) \in G \times G \mid x y=y x\}| /\left(|G|^{2}\right)$. After that, many studies have been developed to determine some bounds for this degree. For example, Gustafson [3] and MacHale [4] proved independently in 1974 that for a non-abelian finite group $\mathrm{G}$, the commutativity degree $d(G) \leq \frac{5}{8}$. Other upper bounds for commutativity degree in terms of centralizers have been obtained for the dihedral group $D_{n}$ by Omer et al. [5]. A classification of the groups for which the commutativity degree is above 11/32 was given in 1979 by Rusin [6], while in 2001, Lescot [7] classified the finite groups with $d(G) \in\left[\frac{1}{2}, 1\right]$, just to recall only some of the studies on this topic.

The notion of commutativity degree was generalized in different ways later on. For example, the probability that an element of a given subgroup of a finite group commutes with an element of the group was studied in [8], and the probability that the commutator of a pair of elements of a finite group equals a certain given number was investigated in [9]. The notion of the subgroup commutativity degree of finite groups was proposed by Tărnăuceanu [10] as the probability that two subgroups of a given group commute, that is, the probability that the product of two subgroups is again a subgroup.

On the other hand, the concept of commutativity degree can also be studied in other algebraic structures, such as in hypergroups. These are a natural generalization of groups, where the operation is substituted by a hyperoperation, i.e., a function defined on the cartesian product of the support set $H$ with values in $\mathcal{P}^{*}(H)$, the family of all non-empty subsets of $H$. Thus, the result of the combination of two elements from the support set is not just an element, as in the classical algebraic structures, but a nonempty subset of the initial set. The hypergroups were introduced by F. Marty in 1934 with their theoretical meaning: The quotient of a group by any of its normal subgroups is again a 
group; while considering a non-normal subgroup, the quotient may be endowed with a hypergroup structure. The recently published paper by Massouros [11] presents the course of development from the hypergroup, as it was initially defined by F. Marty, to the hypergroups endowed with more axioms, and this is used nowadays. The theory of algebraic hypercompositional structures, particularly the hypergroups and hyperrings, becomes then a flourishing area of Modern Algebra, and in the last years, more and more studies in algebraic geometry [12-14], number theory [15], scheme theory [16], tropical geometry [17], and theory of matroids [18] have highlighted the important role of these structures.

In this paper, in the context of complete hypergroups, we address two correlated problems: the class equation and the commutativity degree. We consider this particular type of hypergroup because, by using their representation theorem, i.e., Theorem 1 , we notice that there exists a strong relationship between groups and complete hypergroups. Any complete hypergroup can be obtained using a group, and vice-versa, any group can be viewed as a complete hypergroup. The structure of our study is as follows. First, in Section 2, we fix the notation and terminology, and we recall the basic definitions related with the class equation and commutativity degree in group theory, as well as those related to complete hypergroups, which will be used in the following. The class equation for complete hypergroups is established in Section 3, while in Section 4, we study the commutativity degree for these hypergroups. In addition, we explain that, in order to compute the number of the elements that commute in a finite complete hypergroup, it is necessary to compute the number of the elements that commute in a group, so the commutativity degree of a group influences the commutativity degree of a complete hypergroup (see Theorem 4). This connection is also clearly described with several examples that show that the commutativity degree of a complete hypergroup depends on the decomposition of the hypergroup. Thus, the finite complete hypergroups of a certain cardinality and constructed with the same finite group can have different commutativity degrees. Finally, we calculate the commutativity degree using the conjugacy classes (see Theorem 5)—the same notion used for determining the class equation. The last section contains the conclusions of our study and some open problems related to it.

\section{Preliminaries}

In this section, we first recall the basic notions and results about the class equation and commutativity degree in group theory. Most of them are gathered in the M.A. Thesis of Ref. [19]. Secondly, we briefly present a short review of complete hypergroups, since in the next sections, we will determine the class equation and commutativity degree for such hypergroups. For more details regarding the theory of hypergroups, the reader is refereed to the fundamental books of Refs. [20-22].

\subsection{Class Equation and Commutativity Degree for Groups}

Let $(G, \cdot)$ be a group and $Z(G)=\{a \in G \mid a b=b a, \forall b \in G\}$ be the center of the group $G$.

Definition 1. Ref. [19] We say that two elements $a$ and $b$ are conjugated in $G$, denoted here by $a \sim_{G} b$, if there exists $g \in G$ such that $a=g^{-1} b g$.

The relation $\sim_{G}$ is an equivalence relation, and the equivalence class of each element $a \in G$, i.e., $[a]=\left\{b \in G \mid \exists g \in G: b=g a g^{-1}\right\}$, is called the conjugacy class of $a$. For a finite group $G$, denote by $k(G)$ the number of distinct conjugacy classes of $G$, i.e., $G=\bigcup_{i=1}^{k(G)}\left[x_{i}\right]$. We may recall now the famous class equation in group theory:

$$
|G|=|Z(G)|+\sum_{i=|Z(G)|+1}^{k(G)}\left|\left[x_{i}\right]\right| .
$$


The class equation can be related to another important notion in group theory, one of commutativity degree, which represents the probability that two elements of a group commute [3]. It is defined as follows:

$$
d(G)=\frac{\left|\left\{(a, b) \in G^{2} \mid a \cdot b=b \cdot a\right\}\right|}{|G|^{2}} .
$$

Let us enumerate some properties of the commutativity degree $d(G)[19]$ :

1. $0<d(G) \leq 1$ for any finite group $G$;

2. $d(G)=1$ if and only if $G$ is an abelian group;

3. $d(G) \leq d(H) d(G / H)$ for any finite group $G$ and $H$, a normal subgroup of $G$.

4. The function $d(G)$ is multiplicative, i.e., $d\left(G_{1} \times G_{2}\right)=d\left(G_{1}\right) d\left(G_{2}\right)$, for any two finite groups $G_{1}$ and $G_{2}$.

5. $d(G)=\frac{k(G)}{|G|}$.

\subsection{Complete Hypergroups}

Let $(H, \circ)$ be a hypergroupoid, that is, a nonempty set $H$ endowed with a multi-valued operation $\circ: H \times H \longrightarrow \mathcal{P}^{*}(H)$ (also called hyperoperation), where by $\mathcal{P}^{*}(H)$, we denote the family of nonempty subsets of $H$. For any nonempty subsets $A, B \subseteq H$, we denote

$$
A \circ B=\bigcup_{a \in A, b \in B}(a \circ b),
$$

and thus, if $B$ contains only one element $b$, we simply write $A \circ b$ instead of $A \circ\{b\}$. Now, the reproduction axiom and associativity have sense and they appear in the definition of the following hypercompositional structures:

(i) A semihypergroup is an associative hypergroupoid ( $H, \circ)$, i.e., for all $a, b, c \in H,(a \circ b) \circ c=$ $a \circ(b \circ c)$.

(ii) A quasihypergroup is a hypergroupoid $(H, o)$ that satisfies the reproduction axiom: for all $a \in H$, $H \circ a=a \circ H=H$.

(iii) A hypergroup is a semihypergroup that is also a quasihypergroup.

In any group, there exists only one identity, and each element has a unique inverse. This property does not hold anymore in hypergroups, in the sense that there may exist (or not) more identities, and each element may have more inverses, or none.

An element $e \in H$ is called an identity or unit if, for all $a \in H, a \in a \circ e \cap e \circ a$. An element $a^{\prime} \in H$ is called an inverse of $a \in H$ if there exists an identity $e \in H$, such that $e \in a \circ a^{\prime} \cap a^{\prime} \circ a$. A hypergroup that has at least one identity and has the property that, for each element of $H$, there exists at least one inverse is called regular.

The most natural connection between hypergroups and groups is assured by the fundamental relations because the quotient structure of a hypergroup through a fundamental relation is a group with the same properties of the hypergroup. Let us now recall the definition of one of them, i.e., the $\beta$ relation:

$$
a \beta b \Leftrightarrow \exists n \in \mathbb{N}^{*}, \exists\left(x_{1}, x_{2}, \ldots, x_{n}\right) \in H^{n}:\{a, b\} \subseteq \prod_{i=1}^{n} x_{i} .
$$

If $(H, \circ)$ is a hypergroup, then $\beta$ is an equivalence relation, and the quotient $H / \beta$ is a group. In addition, let $\varphi_{H}: H \longrightarrow H / \beta$ be the canonical projection. The heart of a hypergroup $H$ is the set $\omega_{H}=\left\{x \in H \mid \varphi_{H}(x)=1\right\}$, where 1 is the identity of the group $H / \beta$. 
Let $(H, \circ)$ be a semihypergroup and let $A$ be a nonempty subset of $H$. We say that $A$ is a complete part of $H$ if the following implication holds:

$$
\forall n \in \mathbb{N}^{*}, \forall\left(x_{1}, \ldots, x_{n}\right) \in H^{n}, \prod_{i=1}^{n} x_{i} \cap A \neq \varnothing \Rightarrow \prod_{i=1}^{n} x_{i} \subseteq A .
$$

Moreover, the complete closure of $A$ in $H$, denoted by $\mathcal{C}(A)$, is the intersection of all complete parts of $H$ that contain $A$. It can be characterized using the heart of a hypergroup with $\mathcal{C}(A)=A \circ \omega_{H}=$ $\omega_{H} \circ A=\bigcup_{a \in A} \beta(a)$.

Now, we have all elements for defining the complete hypergroups. Their definition is based on the concept of a complete part. A hypergroup $(H, 0)$ is called complete if, for any $x, y \in H$, there is $\mathcal{C}(x \circ y)=x \circ y$. Hence, for any $x, y \in H$ and $a \in x \circ y$, we have $x \circ y=\beta(a)$.

In practice, it is more convenient to use a construction, which is called hereafter called the representation theorem, starting from a given group, as described in the next result.

Theorem 1. A hypergroup $(H, 0)$ is complete if and only if $H$ can be partitioned as $H=\bigcup_{g \in G} A_{g}$, where $G$ and the subsets $A_{g}$ of $H$ satisfy the following conditions:

1. $(G, \cdot)$ is a group.

2. For all $g_{1} \neq g_{2} \in G$, there is $A_{g_{1}} \cap A_{g_{2}}=\varnothing$.

3. If $(a, b) \in A_{g_{1}} \times A_{g_{2}}$, then $a \circ b=A_{g_{1} \cdot g_{2}}$.

It is worth noticing that, for any group $G$, there are several non-isomorphic complete hypergroups of the same cardinality, depending on the cardinalities of the subsets $A_{g}$ with $g \in G$. This property will have a strong influence on the studies presented in the next sections. For more details and the tables of all non-isomorphic complete hypergroups of order less than 6 , see $[23,24]$.

The most important properties of the complete hypergroups are gathered in the following result.

Theorem 2. Let $(H, \circ)$ be a complete hypergroup.

1. The heart $\omega_{H}$ is the set of all identities of $H$, and thus, $\omega_{H}=A_{e}$, where $e$ is the identity of the group $G$ that appears in the representation theorem of $H$.

2. $H$ is a reversible and regular hypergroup.

\section{The Class Equation for Complete Hypergroups}

The aim of this section is to establish, similarly to in group theory, the class equation for finite complete hypergroups based on the notion of conjugation. The starting point is the representation theorem (Theorem 1) for complete hypergroups, which assures a strong connection between complete hypergroups and groups.

First, we define the conjugation relation between two elements in an arbitrary hypergroup.

Definition 2. Let $(H, \circ)$ be a hypergroup. We say that two elements $a$ and $b$ in $H$ are conjugated, denoted here by $a \sim_{H} b$, if there exists $c \in H$ such that

$$
\mathcal{C}(c \circ a) \cap \mathcal{C}(b \circ c) \neq \varnothing
$$

Since in a complete hypergroup $(H, \circ)$, for any two elements $a, b \in H$, we have $\mathcal{C}(a \circ b)=a \circ b$, then, based on Theorem 1, we may reformulate Definition 2 as follows. 
Definition 3. Let $(H, \circ)$ be a complete hypergroup. We say that the elements $a$ and $b$ are conjugated, denoted here by $a \sim_{H} b$, if there exists $c \in H$ such that

$$
c \circ a=b \circ c
$$

Proposition 1. The relation $\sim_{H}$ is an equivalence relation on the complete hypergroup $H$.

Proof. It is clear that the relation is reflexive, since for $a \in H$, we have $a \circ a=a \circ a$, meaning that $a \sim_{H} a$.

Let us prove now that the relation $\sim_{H}$ is symmetric, and suppose that $a \sim_{H} b$. It follows that there exists $c \in H$ such that $c \circ a=b \circ c$. Since $(H, \circ)$ is a complete hypergroup, there exist (and they are unique) the elements $g_{1}, g_{2}, h \in G$ such that $a \in A_{g_{1}}, b \in A_{g_{2}}$ and $c \in A_{h}$. Therefore, it follows that $A_{h \cdot g_{1}}=A_{g_{2} \cdot h}$, and based on the second condition of Theorem 1, we state that $h g_{1}=g_{2} h$, meaning that $g_{1}=h^{-1} g_{2} h$. Then, $h^{-1} g_{2}=g_{1} h^{-1}$, implying that $A_{h^{-1} \cdot g_{2}}=A_{g_{1} \cdot h^{-1}}$. Thereby, there exists $c^{\prime} \in A_{h^{-1}}$ such that $c^{\prime} \circ b=a \circ c^{\prime}$. This is equivalent with $b \sim_{H} a$, so the relation $\sim_{H}$ is symmetric.

For proving the transitivity, take $a, b, c \in H$, such that $a \sim_{H} b$ and $b \sim_{H} c$. Similarly to proving the symmetry, there exist (and they are unique) the elements $g_{1}, g_{2}, g_{3} \in G$ such that $a \in A_{g_{1}}, b \in A_{g_{2}}$ and $c \in A_{g_{3}}$, and the unique elements $h, k \in G$ such that $h g_{1}=g_{2} h$ and $k g_{2}=g_{3} k$, leading to the equalities $k h g_{1}=k g_{2} h=g_{3} k h$, which mean that $a \sim_{H} c$, concluding the transitivity.

Therefore, $\sim_{H}$ is an equivalence relation.

Based on this proof, a linkage between the conjugated elements in a complete hypergroup and the corresponding conjugated elements in the associated group that appears in the representation of the complete hypergroup can be stated as follows.

Proposition 2. If $(H, \circ)$ is a complete hypergroup obtained from the group $G$, then $a \sim_{H} b$ if and only if $g_{1} \sim \sim_{g_{2}}$, where $a \in A_{g_{1}}$ and $b \in A_{g_{2}}$.

Now, we are ready to define the conjugacy class $[a]$ of an element $a$ of a complete hypergroup $(H, \circ)$ as

$$
[a]=\{b \in H \mid \exists c \in H: c \circ a=b \circ c\} .
$$

It follows very easily that the number of the distinct conjugacy classes in the complete hypergroup $H$ is the same as the number of the distinct conjugacy classes in the associated group, i.e., $k(G)=k(H)$.

Proposition 3. If $(H, \circ)$ is a finite complete hypergroup and $a \in H$, then

$$
|[a]|=\sum_{g_{i} \sim \sim_{G} g}\left|A_{g_{i}}\right|
$$

where $a \in A_{g}$ and $g \in G$.

Proof. In a complete hypergroup $H$, for any $a \in H$, there exists a unique $g \in G$ such that $a \in A_{g}$.

According to Proposition 2 and using the definition of the conjugacy class, we observe that

$$
[a]=\bigcup_{g_{i} \sim \sim_{G} g} A_{g_{i}}
$$

Considering the cardinalities and using the fact that $A_{g_{i}} \cap A_{g_{j}}=\varnothing$, for any $g_{i} \neq g_{j} \in G, i \neq j$, the conclusion immediately follows.

We can state now the main result of this section, namely the class equation for finite complete hypergroups. 
Theorem 3. Let $(H, \circ)$ be a finite complete hypergroup. The following equation holds:

$$
|H|=\left|\omega_{H}\right|+\sum_{a \notin \omega_{H}}|[a]|
$$

Proof. Let $G$ be a finite group of cardinality $n, n \in \mathbb{N}, n \geq 2$, that appears in the representation theorem of the complete hypergroup $H$. We have $\omega_{H}=A_{e}$, where $e$ is the neutral element of the group G. Hence, the complete hypergroup $H$ could be written as

$$
H=A_{e} \cup\left(\bigcup_{g \neq e} A_{g}\right)=\omega_{H} \cup\left(\bigcup_{g \neq e} A_{g}\right)
$$

where the union is a disjoint one.

We intend to check that $\bigcup_{g \neq e} A_{g}=\bigcup_{a \notin \omega_{H}}[a]$.

Let $x \in \bigcup_{a \notin \omega_{H}}[a]$. Then, there exists $a_{0} \notin \omega_{H}$ such that $x \in\left[a_{0}\right]$. Hence, there exists $g \in G, g \neq e$ such that $a_{0} \in A_{g}$. Since $\left[a_{0}\right]=\bigcup_{g_{i} \sim G g} A_{g_{i}}$, it follows that $x \in \bigcup_{g \neq e} A_{g}$. So, $\bigcup_{a \notin \omega_{H}}[a] \subseteq \bigcup_{g \neq e} A_{g}$.

Conversely, from the reflexivity of the relation $\sim_{H}$, it follows that $a \in[a]$. Therefore, $\bigcup_{a \in H} a \subseteq \bigcup_{a \in H}[a]$, and hence $\bigcup_{g \neq e} A_{g} \subseteq \bigcup_{a \notin \omega_{H}}[a]$. Now, the equality is proved.

So, $H$ can be written as a disjoint union as $H=\omega_{H} \cup\left(\bigcup_{a \notin \omega_{H}}[a]\right)$. This implies that

$$
|H|=\left|\omega_{H}\right|+\sum_{a \notin \omega_{H}}|[a]|
$$

Notice that, if the group $G$ is abelian, then the conjugacy class of an element from $H$ is the entire set to which it belongs.

Proposition 4. If $(H, \circ)$ is a complete hypergroup and the group that appears in the representation of $H$ is abelian, then $[a]=A_{g}$, where $a \in A_{g}$.

Proof. We easily check this assertion by using the definition of the conjugacy class $[a]=$ $\{b \in H \mid \exists c \in H: c \circ a=b \circ c\}$. Thus, there exist, and they are unique, the elements $g_{1}, g_{2}$, and $g_{3}$ in $G$ such that $a \in A_{g_{1}}, b \in A_{g_{2}}$, and $c \in A_{g_{3}}$. Thereby, $A_{g_{3}} \cdot g_{1}=A_{g_{2}} \cdot g_{3}$, which implies that $g_{3} g_{1}=g_{2} g_{3}=g_{3} g_{2}$ by the commutativity of $G$, meaning that $g_{1}=g_{2}$. So, we conclude that $b \in A_{g_{1}}$, i.e., $[a]=A_{g_{1}}$.

In the following, we will present an example to highlight the class equation defined above.

Example 1. Let $G=<\rho, \sigma \mid \rho^{3}=\sigma^{2}=(\sigma \rho)^{2}=e>=\left\{e, \rho, \rho^{2}, \sigma, \rho \sigma, \rho^{2} \sigma\right\}$ be the dihedral group $D_{6}$ of order 6 and let $(H, \circ)$ be a proper complete hypergroup of order 7 constructed with the group $G$. According to the representation theorem of the complete hypergroups, we may construct more of such non-isomorphic hypergroups depending on the cardinalities of the subsets $A_{g}$ with $g \in G$ that partition the hypergroup. In every case, five sets $A_{g}$ will contain one element, while the sixth one will contain two elements (this is the unique possibility to decompose the number 7 as a sum of 6 natural numbers). Moreover, the conjugacy classes of the elements of the groups $G=D_{6}$ are $[e]=\{e\},[\rho]=\left\{\rho, \rho^{2}\right\}$, and $[\sigma]=\left\{\sigma, \rho \sigma, \rho^{2} \sigma\right\}$.

Let $H=\left\{a_{0}, a_{1}, a_{2}, a_{3}, a_{4}, a_{5}, a_{6}\right\}$. We analyze the cases:

Case I. Set $A_{e}=\left\{a_{0}\right\}, A_{\rho}=\left\{a_{1}\right\}, A_{\rho^{2}}=\left\{a_{2}, a_{3}\right\}, A_{\sigma}=\left\{a_{4}\right\}, A_{\rho \sigma}=\left\{a_{5}\right\}, A_{\rho^{2} \sigma}=\left\{a_{6}\right\}$. 
For this representation, according to Equation (5), it results that

$$
\omega_{H}=A_{e},\left[a_{1}\right]=\left\{a_{1}, a_{2}, a_{3}\right\},\left[a_{4}\right]=\left\{a_{4}, a_{5}, a_{6}\right\}
$$

Therefore, the formula of the class equation gives

$$
|H|=\left|\omega_{H}\right|+\left|\left[a_{1}\right]\right|+\left|\left[a_{4}\right]\right|
$$

equivalently with $7=1+3+3$.

Case II. Set now $A_{e}=\left\{a_{0}, a_{1}\right\}, A_{\rho}=\left\{a_{2}\right\}, A_{\rho^{2}}=\left\{a_{3}\right\}, A_{\sigma}=\left\{a_{4}\right\}, A_{\rho \sigma}=\left\{a_{5}\right\}, A_{\rho^{2} \sigma}=\left\{a_{6}\right\}$. According to Equation (5), it follows that

$$
\omega_{H}=A_{e},\left[a_{2}\right]=\left\{a_{2}, a_{3}\right\},\left[a_{4}\right]=\left\{a_{4}, a_{5}, a_{6}\right\} .
$$

Hence, the class equation says $|H|=\left|\omega_{H}\right|+\left|\left[a_{2}\right]\right|+\left|\left[a_{4}\right]\right|$, meaning that $7=2+2+3$.

Case III. Take $A_{e}=\left\{a_{0}\right\}, A_{\rho}=\left\{a_{1}\right\}, A_{\rho^{2}}=\left\{a_{2}\right\}, A_{\sigma}=\left\{a_{3}, a_{4}\right\}, A_{\rho \sigma}=\left\{a_{5}\right\}, A_{\rho^{2} \sigma}=\left\{a_{6}\right\}$.

Similarly to the previous cases, it follows that

$$
\omega_{H}=A_{e},\left[a_{1}\right]=\left\{a_{1}, a_{2}\right\},\left[a_{3}\right]=\left\{a_{3}, a_{4}, a_{5}, a_{6}\right\}
$$

Consequently, the class equation gives $|H|=\left|\omega_{H}\right|+\left|\left[a_{1}\right]\right|+\mid\left[a_{3}\right]$, or $7=1+2+4$.

All the other cases are similar to the previous three.

\section{Commutativity Degree of Complete Hypergroups}

The aim of this section is to define the commutativity degree of a hypergroup and to study it in depth for complete hypergroups because of their strong connection with groups. As already mentioned in the preliminaries, the commutativity degree of a finite group is the probability that two arbitrary elements of the group commute. Explicitly, if we denote by

$$
c(G)=\left\{(x, y) \in G^{2} \mid x \cdot y=y \cdot x\right\},
$$

then the commutativity degree can be expressed, as proved in [19], by

$$
d(G)=\frac{|c(G)|}{|G|^{2}}
$$

In a similar way we introduce the commutativity degree for hypergroups. Let $(H, 0)$ be a finite hypergroup. Define the commutativity degree by the formula

$$
d(H)=\frac{\left|\left\{(a, b) \in H^{2} \mid a \circ b=b \circ a\right\}\right|}{|H|^{2}} .
$$

Since any complete hypergroup is constructed with use of a group, we intend to study the commutativity degree of complete hypergroups and find a relationship with the commutativity degree of the corresponding group.

According to the representation theorem for complete hypergroups, i.e., Theorem 1, any finite complete hypergroup $(H, \circ)$ of cardinality $m$ can be represented as $H=\bigcup_{g \in G} A_{g}$, where for any element $g$ of a finite group $(G, \cdot)$ of cardinality $n$, the subsets $A_{g}$ of $H$ satisfy the relations:

i) For any $g_{1} \neq g_{2} \in G$, we have $A_{g_{1}} \cap A_{g_{2}}=\varnothing$;

ii) If $(a, b) \in A_{g_{1}} \times A_{g_{2}}$, then $a \circ b=A_{g_{1} \cdot g_{2}}$. 
Based on this property, the formula of the commutativity degree of a complete hypergroup can be written as

$$
\begin{aligned}
d(H) & =\frac{\left|\left\{(a, b) \mid \exists g_{i}, g_{j} \in G, a \in A_{g_{i}}, b \in A_{g_{j}}, A_{g_{i} g_{j}}=A_{g_{j} g_{i}}\right\}\right|}{|H|^{2}} \\
& =\frac{\left|\left\{(a, b) \mid \exists g_{i}, g_{j} \in G, a \in A_{g_{i}}, b \in A_{g_{j}}, g_{i} g_{j}=g_{j} g_{i}\right\}\right|}{|H|^{2}} .
\end{aligned}
$$

So, we immediately notice a relationship between the pair of the elements commuting in the complete hypergroup and the pair of the elements commuting in the group. This suggests us that there may exist a connection between $d(H)$ and $d(G)$ and in the following, which we aim to find out.

First, we will present a method, which will be described in several steps, for counting the number of the pairs $(a, b) \in H^{2}$ that commute.

Let us fix some notations. For an arbitrary set $S$, we denote its cardinality by $|S|$. Consider the complete hypergroup $(H, \circ)$ with $|H|=m$ and the group $(G, \cdot)$ that appears in the representation of $H$ with $|G|=n$, where $m, n \in \mathbb{N}, m, n \geq 2$. Since we consider only proper hypergroups, we also have the restriction $m>n$. In addition, for any $i \in\{1,2, \ldots, n\}$, take $\left|A_{g_{i}}\right|=m_{i}$, and then $\sum_{i=1}^{n} m_{i}=m$, where $A_{g_{i}}$ are the subsets that appear in the decomposition of $H$, as in Theorem 1.

The main idea of this method is to emphasize the sets $A_{g_{i}}$ that contain one element, so $m_{i}=1$. Then, the remaining sets that contain more than one element, and thus have $m_{i}>1$, will be written as a union of another two their subsets: The first one contains only one element, and the second one contains all the other elements. By doing this, we can count the number of the elements that commute and belong to a singleton set as $|c(G)|=d(G) \cdot|G|^{2}$.

Let us start with a general form of the sets $A_{g_{i}}, i=1,2, \ldots, n$.

$$
\begin{aligned}
A_{g_{1}} & =\left\{x_{1}, \ldots, x_{m_{1}}\right\}=\left\{x_{1}\right\} \cup\left\{x_{2}, \ldots, x_{m_{1}}\right\} \\
A_{g_{2}} & =\left\{x_{m_{1}+1}, \ldots, x_{m_{2}}\right\}=\left\{x_{m_{1}+1}\right\} \cup\left\{x_{m_{1}+2}, \ldots, x_{m_{2}}\right\} \\
& \vdots \\
A_{g_{n}} & =\left\{x_{m_{n-1}+1}, \ldots, x_{n}\right\}=\left\{x_{m_{n-1}+1}\right\} \cup\left\{x_{m_{n-1}+2}, \ldots, x_{n}\right\} .
\end{aligned}
$$

$1^{\circ}$ Step. We count that pairs of elements that commute and belong to sets with only one element. Since we have the equivalences:

$$
\begin{aligned}
x_{1} \circ x_{m_{i}+1} & =x_{m_{i}+1} \circ x_{1} \Leftrightarrow g_{1} g_{i+1}=g_{i+1} g_{1}, \text { for } i=1,2, \ldots, n-1 \\
x_{m_{i}+1} \circ x_{m_{j}+1} & =x_{m_{j}+1} \circ x_{m_{i}+1} \Leftrightarrow g_{i+1} g_{j+1}=g_{j+1} g_{i+1}, i, j=1, \ldots, n-1,
\end{aligned}
$$

we count in this step

$$
|c(G)|=d(G) \cdot|G|^{2}
$$

pairs of elements that commute.

$2^{\circ}$ Step. It is clear that the elements contained in the same set $A_{g_{i}}$ commute between them because their commutativity means $g_{i} \cdot g_{i}=g_{i} \cdot g_{i}$, which is always true. Each element of the set $A_{g_{i}}$ commutes with itself, meaning that we count $m_{i}$ elements. Then, the number of different elements that commute between them is equal to

$$
A_{m_{i}}^{2}=\frac{m_{i} !}{\left(m_{i}-2\right) !}=\left(m_{i}-1\right) m_{i}
$$


Therefore, the number of the elements from the set $A_{g_{i}}$ that commute between them and were not counted at the $1^{\circ}$ Step is $\left(m_{i}-1\right) m_{i}+m_{i}-1=m_{i}^{2}-1$. Since we have $n$ different sets $A_{g_{i}}$, at this step, we count

$$
\sum_{i=1}^{n}\left(m_{i}^{2}-1\right)=\sum_{i=1}^{n} m_{i}^{2}-n
$$

pairs of elements that commute.

$3^{\circ}$ Step. Since all the elements of $G$ commute with the identity $e$, it follows that $a \circ b=b \circ a$ for any $a \in A_{e}, b \in A_{g_{i}}$, and $g_{i} \neq e$. Now, if we take $g_{1}=e$, the number of the ordered pairs of elements from $A_{g_{1}}$ and $A_{g_{2}}$ commuting are $2 m_{1} m_{2}$, but the pairs $\left(x_{1}, x_{m_{1}+1}\right)$ and $\left(x_{m_{1}+1}, x_{1}\right)$ were already counted in the first step. So, we have only $2 m_{1} \cdot m_{2}-2$ pairs. Repeating this procedure for all sets $A_{i}$, we calculate

$$
2\left(m_{2} m_{1}-1\right)+2\left(m_{3} m_{1}-1\right)+\ldots+2\left(m_{n} m_{1}-1\right)=2 \sum_{i=2}^{n}\left(m_{i} m_{1}-1\right)
$$

pairs of elements of this step.

$4^{\circ}$ Step. It remains to consider the pairs of elements from $A_{g_{i}}$ and $A_{g_{j}}$ with $g_{i} g_{j}=g_{j} g_{i}, i \neq j$, $i, j>1$, which were not considered in the first step. It is clear that their number is

$$
2 \sum_{\substack{1<i<j \\ g_{i} g_{j}=g_{j} g_{i}}}\left(m_{i} m_{j}-1\right) \stackrel{\text { not }}{=} c(i, j)
$$

Combining the results of all four cases, we conclude that the commutativity degree of $H$ is

$$
d(H)=\frac{|c(G)|+\sum_{i=1}^{n} m_{i}^{2}-n+2 \sum_{i=2}^{n}\left(m_{i} m_{1}-1\right)+c(i, j)}{|H|^{2}} .
$$

After making some simple computations, we find

$$
d(H)=d(G)\left(\frac{n}{m}\right)^{2}+\frac{\sum_{i=1}^{n} m_{i}^{2}+2 m_{1}\left(m-m_{1}\right)-3 n+2+c(i, j)}{m^{2}} .
$$

The method that we presented before suggests the following result.

Theorem 4. Let $H=\bigcup_{i=1}^{n} A_{g_{i}}$ be a complete hypergroup of cardinality $m$, where $G=\left\{g_{1}, g_{2}, \ldots, g_{m}\right\}$ is the group that appears in the representation of $H$. For any $i=1, \ldots, n$, denote $\left|A_{g_{i}}\right|=m_{i}$. Then, the commutativity degree of $H$ has the formula

$$
d(H)=d(G)\left(\frac{n}{m}\right)^{2}+\frac{\sum_{i=1}^{n} m_{i}^{2}+2 m_{1}\left(m-m_{1}\right)-3 n+2+c(i, j)}{m^{2}},
$$

where $c(i, j)=2 \sum_{\substack{1<i<j \\ g_{i} g_{j}=g_{j} g_{i}}}\left(m_{i} m_{j}-1\right)$, for any $i \neq j \neq 1$ in $\{2, \ldots, n\}$.

Remark 1. If $n=m$, i.e., the complete hypergroup $H$ coincides with the group $G$, we have $m_{i}=1$ for any $i \in\{1, \ldots, n\}$, and thus, based on Formula (13), $c(i, j)=0$. Substituting this into (14), we get

$$
d(H)=d(G)\left(\frac{m}{m}\right)^{2}+\frac{m+2(m-1)-3 m+2+0}{m^{2}}=d(G),
$$

which confirms the validity of the formula. 
In the following example, we will calculate the commutativity degree of some complete hypergroups $H$, of the same cardinality, $m=7$, obtained from the same group $G=D_{6}$, i.e., the dihedral group of order 6, but with different decompositions. More precisely, the cardinalities of the subsets $A_{g}$ with $g \in G$ that partition the hypergroup are different, as we presented in Example 1.

Example 2. Let $(H, \circ)$ be a complete hypergroup of order 7 constructed from the group $D_{6}$, for which we know that $d(G)=\frac{1}{2}[19]$.

I Case. Consider that we have the following decomposition of $H$ :

$A_{e}=\left\{a_{0}, A_{\rho}=\left\{a_{1}\right\}, A_{\rho^{2}}=\left\{a_{2}, a_{3}\right\}, A_{\sigma}=\left\{a_{4}\right\}, A_{\rho \sigma}=\left\{a_{5}\right\}, A_{\rho^{2} \sigma}=\left\{a_{6}\right\}\right.$. Then, using formula (14), we obtain

$$
d(H)=\frac{\frac{1}{2} \cdot 6^{2}+5 \cdot 1^{2}+2^{2}+2(7-1)-3 \cdot 6+2+2(2 \cdot 1-1)}{7^{2}}=\frac{25}{49}>d(G) .
$$

II Case. Consider that we have the following decomposition of $H: A_{e}=\left\{a_{0}, a_{1}\right\}, A_{\rho}=\left\{a_{2}\right\}, A_{\rho^{2}}=$ $\left\{a_{3}\right\}, A_{\sigma}=\left\{a_{4}\right\}, A_{\rho \sigma}=\left\{a_{5}\right\}, A_{\rho^{2} \sigma}=\left\{a_{6}\right\}$. Again, using formula (14), we get $d(H)=\frac{31}{49}>\frac{25}{49}>d(G)$.

III Case. For the following representation of $H$, where $A_{e}=\left\{a_{0}\right\}, A_{\rho}=\left\{a_{1}\right\}, A_{\rho^{2}}=\left\{a_{2}\right\}, A_{\sigma}=$ $\left\{a_{3}, a_{4}\right\}, A_{\rho \sigma}=\left\{a_{5}\right\}, A_{\rho^{2} \sigma}=\left\{a_{6}\right\}$, we obtain $d(H)=\frac{23}{49}<d(G)$.

Concluding, for this kind of complete hypergroup, we obtain three different values of $d(H)$ and exactly

$$
d(H)=\left\{\frac{23}{49}, \frac{25}{49}, \frac{31}{49}\right\} .
$$

In the following, we will express the commutativity degree $d(H)$ of a complete hypergroup as a formula involving the conjugacy classes of the elements of $H$. In group theory, it is known that the commutativity degree of a finite group $G$ can be expressed as $d(G)=\frac{k(G)}{|G|}$, where $k(G)$ represents the number of the distinct conjugacy classes of the elements of $G$.

Now, we will explain how and why this formula changes in the class of hypergroups.

First of all, we will recall some results concerning groups. For any element $g$ of a group $G$, we denote by $[g]$ the conjugacy class of g, i.e.

$$
[g]=\left\{h \in G \mid \exists a \in G: h=a g a^{-1}\right\},
$$

and by $C_{G}(g)$ the centralizer of the element $g$ in $G$, i.e., $C_{G}(g)=\{h \in G \mid g h=h g\}$, which is a subgroup of $G$.

Then, we have

$$
|[g]|=\left[G: C_{G}(g)\right]=\frac{|G|}{\left|C_{G}(g)\right|},
$$

meaning that the product $|[g]| \cdot\left|C_{G}(g)\right|$ is constant, or in other words, for any $h \in[g]$, it is $\left|C_{G}(h)\right|=$ $\left|C_{G}(g)\right|$.

This property does not hold anymore for complete hypergroups, as we can notice for the hypergroups described in Example 2.

For an arbitrary hypergroup $H$, the centralizer $C_{H}(x)$ of an element $x$ in $H$ is defined as

$$
C_{H}(x)=\{y \in H \mid C(x \circ y)=C(y \circ x)\},
$$


where in the particular case of the complete hypergroups, the complete closure $C(x \circ y)$ is equal to $x \circ y$. So, for a complete hypergroup $(H, 0)$ with the representation $H=\bigcup_{g \in G} A_{g}$, for any $x \in H$, there exists a unique $g_{x} \in G$ such that $x \in A_{g_{x}}$ and thereby:

$$
\begin{gathered}
C_{H}(x)=\{y \in H \mid x \circ y=y \circ x\}=\left\{y \in A_{g_{y}} \mid A_{g_{x} \cdot g_{y}}=A_{g_{y} \cdot g_{x}}\right\}= \\
=\left\{y \in A_{g_{y}} \mid g_{x} \cdot g_{y}=g_{y} \cdot g_{x}\right\}=\left\{y \in A_{g_{y}} \mid g_{y} \in C_{G}\left(g_{x}\right)\right\}=\bigcup_{g \in C_{G}\left(g_{x}\right)} A_{g},
\end{gathered}
$$

where we have a disjoint union.

It follows that

$$
\left|C_{H}(x)\right|=\sum_{g \in C_{G}\left(g_{x}\right)}\left|A_{g}\right|
$$

Theorem 5. If $(H, \circ)$ is a finite complete hypergroup represented by the group $G$ and $\left|C_{H}(x)\right|=\left|C_{H}(y)\right|$ for any $y \in[x]$, then the commutativity degree of $H$ is

$$
d(H)=\frac{\sum_{i=1}^{k(H)}\left|\left[x_{i}\right]\right| \cdot\left|C_{H}\left(x_{i}\right)\right|}{|H|^{2}}
$$

where $k(H)$ is the number of the distinct conjugacy classes $\left[x_{i}\right]$ of the elements of $H$, and thus, $H=\bigcup_{i=1}^{k(H)}\left[x_{i}\right]$.

Proof. We know already from the previous section that $k(G)=k(H)$. First, we will determine the cardinality of the centralizer $c(H)$ of the complete hypergroup $H=\bigcup_{i=1}^{k(H)}\left[x_{i}\right]$. Therefore,

$$
\begin{aligned}
& c(H)=\left\{(a, b) \in H^{2} \mid a \circ b=b \circ a\right\}=\left\{(a, b) \in H^{2} \mid b \in C_{H}(a), a \in \bigcup_{i=1}^{k(H)}\left[x_{i}\right]\right\} \\
& =\left\{(a, b) \in H^{2} \mid b \in C_{H}(a), a \in\left[x_{1}\right]\right\} \bigcup\left\{(a, b) \in H^{2} \mid b \in C_{H}(a), a \in\left[x_{2}\right]\right\} \\
& \cup \ldots \cup\left\{(a, b) \in H^{2} \mid b \in C_{H}(a), a \in\left[x_{k(H)}\right]\right\} \\
& =\bigcup_{i=1}^{k(H)} E_{i},
\end{aligned}
$$

where $E_{i}=\left\{(a, b) \in H^{2} \mid b \in C_{H}(a), a \in\left[x_{i}\right]\right\}$, for $i=\{1, \ldots, k(H)\}$. Since two distinct conjugacy classes are disjoint, it follows that the set $E_{i}$ is disjoint, and therefore, $|c(H)|=\sum_{i=1}^{k(H)}\left|E_{i}\right|$.

Now let us determine the cardinality of each set $E_{i}$. Denote

$$
\left[x_{i}\right]=\left\{x_{i_{1}}, x_{i_{2}}, \ldots, x_{i_{p}}\right\}, p \in \mathbb{N} \backslash\{0\} .
$$

Then, for $a=x_{i_{j}}$ and $b \in C_{H}(a)$, we obtain $\left|\left\{\left(x_{i_{j}}, b\right) \in H^{2} \mid b \in C_{H}\left(x_{i_{j}}\right)\right\}\right|=\left|C_{H}\left(x_{i_{j}}\right)\right|$ for $1 \leq j \leq p$, implying that

$$
\left|E_{i}\right|=\sum_{j=1}^{p}\left|C_{H}\left(x_{i_{j}}\right)\right| .
$$

Now, according to the hypothesis, for any $x_{i_{j}} \in\left[x_{i}\right]$, we have

$$
\left|C_{H}\left(x_{i_{j}}\right)\right|=\left|C_{H}\left(x_{i}\right)\right|,
$$


thereby, $\left|E_{i}\right|=\left|\left[x_{i}\right]\right| \cdot\left|C_{H}\left(x_{i}\right)\right|$, and so

$$
|c(H)|=\sum_{i=1}^{k(H)}\left|\left[x_{i}\right]\right| \cdot\left|C_{H}\left(x_{i}\right)\right|
$$

Based on the definition of the commutativity degree $d(H)$, we conclude that

$$
d(H)=\frac{\sum_{i=1}^{k(H)}\left|\left[x_{i}\right]\right| \cdot\left|C_{H}\left(x_{i}\right)\right|}{|H|^{2}} .
$$

Example 3. Let us continue with Case I of Example 2, when $\left[a_{0}\right]=\left\{a_{0}\right\},\left[a_{1}\right]=\left\{a_{1}, a_{2}, a_{3}\right\}$ and $\left[a_{4}\right]=$ $\left\{a_{4}, a_{5}, a_{6}\right\}$. We check that the condition $\left|C_{H}(x)\right|=\left|C_{H}(y)\right|$, for any $y \in[x]$, holds. First, we determine the centralizer of each element of $H$ and we obtain:

$$
\begin{aligned}
& C_{H}\left(a_{0}\right)=H ; \\
& C_{H}\left(a_{1}\right)=A_{e} \cup A_{\rho} \cup A_{\rho^{2}}=\left\{a_{0}, a_{1}, a_{2}, a_{3}\right\}=C_{H}\left(a_{2}\right)=C_{H}\left(a_{3}\right) ; \\
& C_{H}\left(a_{4}\right)=A_{e} \cup A_{\sigma}=\left\{a_{0}, a_{4}\right\} ; \\
& C_{H}\left(a_{5}\right)=A_{e} \cup A_{\rho \sigma}=\left\{a_{0}, a_{5}\right\} ; \\
& C_{H}\left(a_{6}\right)=A_{e} \cup A_{\rho^{2} \sigma}=\left\{a_{0}, a_{6}\right\} .
\end{aligned}
$$

So, it is clear that the condition in the hypothesis of Theorem 5 holds.

Now let us calculate the cardinalities of the sets $E_{i}$, when

$$
c(H)=\bigcup_{i=1}^{k(H)} E_{i}=\bigcup_{i=1}^{3} E_{i},
$$

with

$$
\begin{aligned}
E_{1} & =\left\{(a, b) \in H^{2} \mid b \in C_{H}(a), a \in\left[a_{0}\right]\right\}=\left\{\left(a_{0}, b\right) \in H^{2} \mid b \in H\right\} ; \\
E_{2} & =\left\{(a, b) \in H^{2} \mid b \in C_{H}(a), a \in\left[a_{1}\right]\right\} \\
& =\bigcup_{i=1}^{3}\left\{\left(a_{i}, b\right) \in H^{2} \mid b \in C_{H}\left(a_{i}\right)\right\} ; \\
E_{3} & =\left\{(a, b) \in H^{2} \mid b \in C_{H}(a), a \in\left[a_{4}\right]\right\} \\
& =\bigcup_{i=4}^{6}\left\{\left(a_{i}, b\right) \in H^{2} \mid b \in C_{H}\left(a_{i}\right)\right\} .
\end{aligned}
$$

Therefore, we have $\left|E_{1}\right|=7,\left|E_{2}\right|=\sum_{i=1}^{3}\left|C_{H}\left(a_{i}\right)\right|=\left|\left[a_{1}\right]\right| \cdot\left|C_{H}\left(a_{1}\right)\right|=3 \cdot 4=12,\left|E_{3}\right|=$ $\sum_{j=4}^{6}\left|C_{H}\left(a_{j}\right)\right|=\left|\left[a_{4}\right]\right| \cdot\left|C_{H}\left(a_{4}\right)\right|=3 \cdot 2=6$, and then, the commutativity degree is

$$
d(H)=\frac{\left|E_{1}\right|+\left|E_{2}\right|+\left|E_{3}\right|}{|H|^{2}}=\frac{7+12+6}{7^{2}}=\frac{25}{49}
$$


which has the same value if we calculate it with the formula (16) in Theorem 5:

$$
d(H)=\frac{\sum_{i=1}^{k(H)}\left|\left[a_{i}\right]\right| \cdot\left|C_{H}\left(a_{i}\right)\right|}{|H|^{2}}=\frac{1 \cdot 7+3 \cdot 4+3 \cdot 2}{7^{2}}=\frac{25}{49} .
$$

In the next example, we will consider the same group $G=D_{6}$ and a complete hypergroup $H$ of the same cardinality 7 constructed from $G$, but with a different partition of the sets $A_{g}$. Here, the condition expressed in Theorem 5 does hold anymore, and the formula (16) is also not true.

Example 4. Consider now the hypergroup in Case III of Example 2, where $\left[a_{0}\right]=\left\{a_{0}\right\},\left[a_{1}\right]=\left\{a_{1}, a_{2}\right\}$, and $\left[a_{3}\right]=\left\{a_{3}, a_{4}, a_{5}, a_{6}\right\}$.

As in Example 3, first, we determine the centralizers of the elements:

$$
\begin{aligned}
& C_{H}\left(a_{0}\right)=H, \\
& C_{H}\left(a_{1}\right)=A_{e} \cup A_{\rho} \cup A_{\rho^{2}}=\left\{a_{0}, a_{1}, a_{2}\right\}, \\
& C_{H}\left(a_{3}\right)=A_{e} \cup A_{\sigma}=\left\{a_{0}, a_{3}, a_{4}\right\}, \\
& C_{H}\left(a_{5}\right)=A_{e} \cup A_{\rho \sigma}=\left\{a_{0}, a_{5}\right\}, \\
& C_{H}\left(a_{6}\right)=A_{e} \cup A_{\rho^{2} \sigma}=\left\{a_{0}, a_{6}\right\} .
\end{aligned}
$$

We notice that $a_{5} \in\left[a_{3}\right]$, but $\left|C_{H}\left(a_{5}\right)\right| \neq\left|C_{H}\left(a_{3}\right)\right|$, so the condition in Theorem 5 does not hold. Now, by calculating $\sum_{x \in H}\left|C_{H}(x)\right|$, we obtain that its value is $1 \cdot 7+2 \cdot 3+2 \cdot 3+2+2=23$, sod $(H)=\frac{23}{49}$, as we calculated in Example 2.

If we apply the formula (16), we get

$$
d(H)=\frac{\left|\left[a_{0}\right]\right| \cdot\left|C_{H}\left(a_{0}\right)\right|+\left|\left[a_{1}\right]\right| \cdot\left|C_{H}\left(a_{1}\right)\right|+\left|\left[a_{3}\right]\right| \cdot\left|C_{H}\left(a_{3}\right)\right|}{|H|^{2}}=\frac{25}{49}
$$

which is not the correct value.

\section{Conclusions}

Any finite group can be partitioned in sets of disjoint conjugacy classes. The class equation states that the cardinality of the group is equal to the sum of the cardinalities of the above-mentioned classes. This result has different applications in group theory; among them, we recall the degree of the commutativity of a group, that is, the probability for two different elements to commute (in a non-commutative group). These two fundamental concepts, i.e., the class equation and commutativity degree, are also related in the hypergroup theory. In this paper, we studied them for complete hypergroups. First, we defined the conjugacy relation on a hypergroup, which is an equivalence relation, proving that, in a complete hypergroup, the number of the distinct conjugacy classes is the same as the number of the distinct conjugacy classes in the group that appears in the construction of the considered complete hypergroup. We then stated the class equation for such hypergroups, which is similar to the class equation for groups, with the difference that the role of the center of the group is played now by the heart of the complete hypergroup. The second part of the paper is dedicated to the study of the commutativity degree of a complete hypergroup. This is the probability that two randomly chosen elements commute in a non-commutative hypergroup. It has been already studied for HX-groups [25] and polygroups [26]. First, we have presented a general method to compute this degree, obtaining a formula that depends on the commutativity degree of the group that appears in the representation theorem of the considered complete hypergroup. It is important to stress the fact that, by using a certain group, more non-isomorphic complete hypergroups of the same cardinality can be constructed. Thus, their commutativity degree is not the same, but if the centralizer of each element in 
a conjugacy class is the same, i.e., $\left|C_{H}(y)\right|=\left|C_{H}(x)\right|$ for any $y \in[x]$, then the commutativity degree of the complete hypergroup $H$ can be expressed using the class equation of $H$ (see Theorem 5). Moreover, it would be interesting to see under which conditions the commutativity degree of the complete hypergroups of a fixed cardinality and constructed from the same group are maximal, or to find a constant $c \in(0,1]$ such that if the commutativity degree is greater than $c$, then the complete hypergroup is commutative. These problems will be investigated in our future work, since the commutativity aspect is an interesting topic in algebra, and is also studied for polynomials, for example, in $[27,28]$.

Author Contributions: Conceptualization, A.C.S. and I.C.; Funding acquisition, I.C.; Investigation, A.C.S. and I.C.; Methodology, A.C.S. and I.C.; Writing-original draft, A.C.S.; Writing-review and editing, I.C. Both authors have read and agreed to the published version of the manuscript.

Funding: The second author acknowledges the financial support from the Slovenian Research Agency (research core funding No. P1-0285).

Conflicts of Interest: The authors declare no conflict of interest.

\section{References}

1. Miller, G. Relative number of non-invariant operators in a group. Prco. Nat. Acad. Sci. USA 1944, 30, $25-28$.

2. Erdős, P.; Turán, P. On Some Problems of a Statistical Group Theory. Acta Math. Acad. Hung. 1968, 19, 413-435.

3. Gustafson, W.H. What is the probability that two group elements commute? Am. Math. Mon. 1974, 80, 1031-1034.

4. MacHale, D. How commutative can a non-commutative group be? Math. Gaz. 1974, 58, 199-202.

5. Omer, S.M.S.; Sarmin, N.H.; Ploradipour, K.; Erfanian, A. The computation of the commutativity degree for dihedral groups in terms of centralizers. Aust. J. Basic Appl. Sci. 2012, 6, 48-52.

6. Rusin, D.J. What is the probability that two elements of a finite group commute? Pac. J. Math. 1979, 82, 237-247.

7. Lescot, P. Central extensions and commutativity degree. Comm. Algebra 2001, 29, 4451-4460.

8. Erfanian, A.; Rezaei, R.; Lescot, P. On the relative commutativity degree of a subgroup of a finite group. Comm. Algebra 2007, 35, 4183-4197.

9. Pournaki, M.R.; Sobhani, R. Probability that the commutator of two group elements is equal to a given element. J. Pure Appl. Algebra 2008, 212, 727-734.

10. Tărnăuceanu, M. Subgroup commutativity degrees of finite groups. J. Algebra 2011, 337, 363-368.

11. Massouros, G.; Massouros, C. Hypercompositional Algebra, Computer Science and Geometry. Mathematics 2020, 8, 1338. [CrossRef]

12. Connes, A.; Consani, C. On the notion of geometry over $\mathbf{F}_{1}$. J. Algebraic Geom. 2011, 20, 525-557. [CrossRef]

13. Jun, J. Algebraic geometry over hyperrings. Adv. Math. 2018, 323, 142-192. [CrossRef]

14. Vahedi, V.; Jafarpour, M.; Cristea, I. Hyperhomographies on Krasner hyperfields. Symmetry 2019, 11, 1442. [CrossRef]

15. Connes, A.; Consani, C. The hyperring of adele classes. J. Number Theory 2011, 131, 159-194. [CrossRef]

16. Jun, J. Hyperstructres of affine algebraic group schemes. J. Number Theory 2016, 167, 336-352. [CrossRef]

17. Viro, O. Hyperfields for tropical geometry I. Hyperfields and dequantization. arXiv 2010, arXiv: 1006.3034.

18. Baker, M.; Bowler, N. Matroids over partial hyperstructures. Adv. Math. 2019, 343, 821-863. [CrossRef]

19. Castelaz, A. Commutativity Degree of Finite Groups. Master's Thesis, Wake Forest University, Winston-Salem, NC, USA, May 2010. Available online: https://bit.ly/2N9Vca7 (accessed on 17 November 2020).

20. Corsini, P. Prolegomena of Hypergroup Theory; Aviani Editore: Tricesimo, Italy, 1993.

21. Corsini, P.; Leoreanu, V. Applications of Hyperstructures Theory; Kluwer Academic Publishers: Boston, MA, USA; Dordrechtt, The Netherlands; London, UK, 2013.

22. Vougiouklis, T. Hyperstructures and Their Representations, Monographs in Mathematics; Hadronic Press: Palm Harbor, FL, USA, 1994.

23. Cristea, I. Complete hypergroups, 1-hypergroups and fuzzy sets. An. St. Univ. Ovidius Constanta Ser. Mat. 2002, 10, 25-38. 
24. Cristea, I.; Hassani Sadrabadi, E.; Davvaz, B. A fuzzy application of the group $\mathbb{Z}_{n}$ to complete hypergroups. Soft Comput. 2020, 24, 3543-3550. [CrossRef]

25. Sonea, A.C. HX-groups associated with the dihedral group $D_{n}$. J. Mult.-Valued Logic Soft Comput. 2019, 33, 11-26.

26. Sonea, A.C. New aspects in polygroup theory. An. St. Univ. Ovidius Constanta Ser. Mat. 2020, 28, $241-254$.

27. Kanel-Belov, A.; Malev, S.; Rowen, L.; Yavich, R. Evaluations of noncommu-tative polynomials on algebras: Methods and problems, and the Lvov-Kaplansky Conjec-ture. SSIGMA 2020, 16, 071.

28. Belov, A.; Bokut, L;. Rowen, L.; Yu, J. The Jacobian conjecture, together with Specht and Burnside-type problems. In Automorphisms in Birational and Affine Geometry (Bellavista Relax Hotel, Levico Terme Trento, Italy, 29 October-3 November 2012); Springer Proceedings in Mathematics and Statistics, 79; Springer: Berlin/Heidelberg, Germany, 2014; pp. 249-285.

Publisher's Note: MDPI stays neutral with regard to jurisdictional claims in published maps and institutional affiliations.

(C) 2020 by the authors. Licensee MDPI, Basel, Switzerland. This article is an open access article distributed under the terms and conditions of the Creative Commons Attribution (CC BY) license (http://creativecommons.org/licenses/by/4.0/). 